\title{
Revista Colombiana de

\section{Stent liberadores de medicamento en enfermedad coronaria prematura en jóvenes con hipercolesterolemia familiar homocigota y trasplante hepático previo}

\author{
Darío Echeverri ${ }^{\mathrm{a}, \mathrm{b}, *}$, Jaime R. Cabrales ${ }^{\mathrm{a}, \mathrm{b}}$, Juan Hernando del Portillo ${ }^{\mathrm{a}, \mathrm{b}}$ y Dairo Rey ${ }^{\mathrm{c}, \mathrm{d}}$
}

a Servicio de Hemodinamia e Intervencionismo Cardiovascular, Fundación Cardiolnfantil-Instituto de Cardiología, Bogotá, Colombia

${ }^{\mathrm{b}}$ Facultad de Medicina-Universidad del Rosario, Bogotá, Colombia

c Cardiología Clínica, Fundación Cardiolnfantil-Instituto de Cardiología, Bogotá, Colombia

d Facultad de Medicina-Universidad El Bosque, Bogotá, Colombia

Recibido el 4 de agosto de 2016; aceptado el 26 de octubre de 2016

Disponible en Internet el 13 de enero de 2017

\author{
PALABRAS CLAVE \\ Hipercolesterolemia \\ familiar; \\ Enfermedad \\ coronaria; \\ Stents liberadores de \\ fármacos; \\ Colesterol LDL
}

\begin{abstract}
Resumen La hipercolesterolemia familiar es una enfermedad genética que se caracteriza por niveles muy elevados de colesterol y lipoproteínas de baja densidad en suero, xantomas tendinosos y aterosclerosis prematura. La forma heterocigota es la más común; alcanza una prevalencia de aproximadamente 1 de cada 300 a 500 personas en el mundo, en tanto que la homocigota, autosómica dominante, es la forma más rara, con una prevalencia de 1 en 1 millón de personas. Esta se caracteriza por hipercolesterolemia severa, que conlleva enfermedad cardiovascular prematura y a menudo no responde al tratamiento tradicional por la falta de receptores para c-LDL funcionales. Los niveles de c-LDL pueden superar seis a diez veces los valores normales, en cuyo caso el trasplante de hígado se ha convertido en el tratamiento de elección para los pacientes que no responden a tratamientos farmacológicos de rutina. Se presentan dos casos con hipercolesterolemia familiar homocigota en jóvenes de 14 y 15 años, con antecedente de trasplante de hígado y enfermedad coronaria severa en vasos principales (descendente anterior y coronaria derecha) a quienes se les hizo implante exitoso de stent liberador de medicamento. (c) 2016 Sociedad Colombiana de Cardiología y Cirugía Cardiovascular. Publicado por Elsevier España, S.L.U. Este es un artículo Open Access bajo la licencia CC BY-NC-ND (http:// creativecommons.org/licenses/by-nc-nd/4.0/).
\end{abstract}

\footnotetext{
- Siguiendo la política de publicación de la revista, este artículo, en el que ha participado como coautor un miembro del Comité editorial, ha superado un estricto proceso de revisión por pares doble ciego y los autores no han tenido ninguna injerencia en la aprobación para su publicación.

* Autor para correspondencia.

Correo electrónico: decheverri@cardioinfantil.org (D. Echeverri).
} 


\section{KEYWORDS}

Familial hypercholesterolemia; Coronary disease; Drug-eluting stents; LDL Cholesterol
Drug-eluting stents in premature coronary disease in young people with homozygous familial hypercholesterolemia and prior liver transplantation

Abstract Familial hypercholesterolemia is a genetic disorder characterised by very high cholesterol and low-density lipoproteins serum levels, tendon xanthomas and premature atherosclerosis. Heterozygous form is the most common, with a prevalence of approximately 1 out of 300 to 500 people worldwide, whereas the homozygous, autosomal dominant, is the rarest form, with a prevalence of 1 out of 1 million people. It is characterised by severe hypercholesterolemia leading to premature cardiovascular disease, and it often does not respond to traditional therapy due to the lack of receptors for functional LDL-c. LDL-c levels can exceed between six and ten times the normal values, in which case liver transplantation has become the treatment of choice for patients who do not respond to routine pharmacological therapies. This study presents two cases of homozygous familial hypercholesterolemia in young patients aged 14 and 15, with prior liver transplantation and severe coronary disease in major vessels (anterior descending artery and right coronary artery) who underwent successful implant of a drug-eluting stent.

(c) 2016 Sociedad Colombiana de Cardiología y Cirugía Cardiovascular. Published by Elsevier España, S.L.U. This is an open access article under the CC BY-NC-ND license (http:// creativecommons.org/licenses/by-nc-nd/4.0/).

\section{Introducción}

Los trastornos del metabolismo de las lipoproteínas, así como las dietas ricas en grasas, la obesidad y la inactividad física, han dado lugar a una epidemia mundial de enfermedad aterosclerótica. La interacción de los trastornos genéticos y adquiridos de las lipoproteínas con estos factores ambientales adversos, predispone a aterosclerosis prematura. En los Estados Unidos, la mortalidad por enfermedad coronaria, principalmente en personas de mediana edad, ha disminuido en un $31 \%$ en la última década; sin embargo, la enfermedad cardiovascular aterosclerótica sigue siendo la causa más común de muerte tanto en hombres como en mujeres. La tasa general de muertes atribuibles a enfermedades cardiovasculares es de 235,5 por 100.000 habitantes y la enfermedad coronaria sola causó 1 de cada 6 muertes en los Estados Unidos en el $2010^{1}$.

La hipercolesterolemia familiar (HF) es una enfermedad genética que se caracteriza por niveles muy elevados de colesterol y lipoproteínas de baja densidad en suero, xantomas tendinosos y aterosclerosis prematura ${ }^{2}$. Más del $85 \%$ de los casos de HF se deben a más de 1.600 mutaciones heredadas en el gen del receptor LDL (R-LDL). Dicha mutación conduce a la captación defectuosa del c-LDL de la sangre ${ }^{3-5}$.

La HF heterocigota es la forma más común de la enfermedad (prevalencia de aproximadamente 1 de cada 300 a 500 personas en todo el mundo y tan alta como 1 de cada 100 personas en algunas poblaciones $)^{3-6}$, mientras que la HF homocigota, autosómica dominante, es la forma más rara (prevalencia de 1 en 1 millón de personas). Algunas poblaciones, como los franco-canadienses, judíos Ashkenazi, libaneses y holandeses de origen africano, están en riesgo más alto para HF debido a un aumento de la prevalencia de mutaciones asociadas a HF heterocigota ${ }^{7-10}$.

La variable homocigota se caracteriza por hipercolesterolemia severa que conduce a enfermedad cardiovascular prematura. Los individuos tienen mayor riesgo de eventos cardiacos como infarto de miocardio y muerte por enfermedad coronaria prematura, especialmente aquellos con formas severas que no han sido tratados ${ }^{11,12}$. Constituye una forma grave y agresiva de la enfermedad, que a menudo no responde al tratamiento tradicional dada la falta de receptores para c-LDL funcionales ${ }^{12,13}$. En general, la edad promedio en el momento del diagnóstico de las manifestaciones cardiovasculares es $20 \operatorname{años}^{12}$. La elevación de los niveles de c-LDL refleja la severidad de la mutación genética. En pacientes con HF heterocigota típicamente se presentan niveles al doble o triple en comparación con individuos sanos (aproximadamente $200-400 \mathrm{mg} / \mathrm{dl}$ ), mientras que los pacientes con HF homocigota tienen niveles de c-LDL que pueden superar seis a diez veces los valores normales $(>600 \mathrm{mg} / \mathrm{dl})^{13}$.

Así mismo, la forma homocigota se asocia con enfermedad coronaria y muerte prematura; de hecho existen varios informes en menores de 17 años de edad que desarrollaron estenosis coronaria severa ${ }^{2,14}$ y estenosis aórtica supravalvular ${ }^{15}$, aumento del grosor de la íntima-media de la carótida y arteria femoral ${ }^{16}$ y xantomas tendinosos causados por el depósito de colesterol en los tendones y la piel, que se observan principalmente en codos, rodillas, tendón de Aquiles, y dorso de manos y pies. Son altamente sugestivos de HF homocigota y son un criterio básico en el diagnóstico clínico de esta enfermedad. En pacientes con HF existen xantomas hasta en el $50 \%$ de los $\operatorname{casos}^{17}$. El colesterol en exceso también se puede depositar en la córnea, dando lugar al arco corneal. Para conocer más a fondo sobre esta patología lo invitamos a consultar el artículo especial de revisión sobre HF publicado por Merchán et al. ${ }^{18}$.

Este artículo obedece a las características no comunes en el tratamiento de pacientes con HF homocigota como lo son la enfermedad aterosclerótica progresiva en adolescentes luego de trasplante hepático exitoso y el control de su perfil lipídico, que obligan a un manejo interdisciplinario complejo, así como a un seguimiento estricto. 


\section{Pacientes y métodos}

Se describe la experiencia de dos pacientes jóvenes que acudieron al Servicio de Cardiología Intervencionista de la institución, en septiembre y octubre de 2013, con características clínicas de HF homocigótica, trasplante hepático y enfermedad coronaria severa, y fueron sometidos a revascularización miocárdica percutánea con implante de stent liberadores de fármaco.

\section{Caso 1}

Hombre de 15 años de edad (tablas 1 y 2), con cuadro de angina de pecho de dos semanas de evolución, deterioro de clase funcional, infarto sin elevación del ST, troponina (+), antecedente de HF homocigota (fig. 1), xantomas cutáneos tuberosos (fig. 2), hiperlipidemia severa (c-LDL hasta de $850 \mathrm{mg} / \mathrm{dl}$ ), hipertensión arterial estadio 1; anomalía de Ebstein y síndrome de Wolff-Parkinson-White ablactado en septiembre de 2011, sometido a trasplante de hígado en junio-2013, y prueba de esfuerzo submáxima positiva para isquemia. Se hallaba en tratamiento farmacológico con ezetimiba $10 \mathrm{mg} /$ día, simvastatina $20 \mathrm{mg}$ /día, amlodipino $5 \mathrm{mg} /$ día, prednisolona $2,5 \mathrm{mg}$ /día y tacrolimus $3 \mathrm{mg} /$ día. La angiografía coronaria reveló enfermedad coronaria severa en arteria descendente anterior proximal y coronaria derecha ostial. Se realizó angioplastia coronaria con implante de stent liberador de medicamento Resolute Integrity ${ }^{\top M}$ (Medtronic Vascular, Santa Rosa, CA) 3,0 $\times 12 \mathrm{~mm}$

Tabla 1 Manifestaciones demográficas, clínicas y paraclínicas de los dos casos con hipercolesterolemia familiar homocigota

\begin{tabular}{|c|c|c|}
\hline & Caso 1 & Caso 2 \\
\hline \multicolumn{3}{|l|}{ Factores demográficos } \\
\hline Edad (años) & 15 & 14 \\
\hline Sexo & M & $\mathrm{F}$ \\
\hline Raza & Hispana & Hispana \\
\hline \multicolumn{3}{|l|}{ Criterios clínicos } \\
\hline Angina (CCS) & II/IV & III/IV \\
\hline Disnea (NYHA) & II/IV & II/IV \\
\hline IMNST & Sí & No \\
\hline \multicolumn{3}{|l|}{ Antecedentes } \\
\hline Hipertensión arterial (estadio) & 1 & No \\
\hline Colesterol total $(\mathrm{mg} / \mathrm{dl})$ & 575 & 850 \\
\hline $\mathrm{c}-\mathrm{LDL}(\mathrm{mg} / \mathrm{dl})$ & 573 & 644 \\
\hline Anomalía de Ebstein & Sí & No \\
\hline Síndrome WPW & Sí - Ablación septal -2011 & No \\
\hline Trasplante hepático & Sí (junio 2013) & Si (septiembre 2013) \\
\hline Estenosis aórtica supravalvular & Sí & No \\
\hline \multicolumn{3}{|l|}{ Examen físico } \\
\hline Peso $(\mathrm{kg})$ & 52 & 47 \\
\hline Talla $(\mathrm{cm})$ & 163 & 150 \\
\hline IMC & 19.6 & 20,8 \\
\hline Presión arterial (mm Hg)- promedio & $121 / 69$ & $100 / 60$ \\
\hline Frecuencia cardiaca (lat/min)- promedio & 72 & 88 \\
\hline Soplo carotídeo & Sí & No \\
\hline Soplo aórtico & Sí & No \\
\hline Xantomas & Sí & Sí \\
\hline Arco corneano & No & Sí \\
\hline Xantelasmas & No & No \\
\hline \multicolumn{3}{|l|}{ Exámenes sanguíneos } \\
\hline Hemoglobina (g/dl) & 14,7 & 11,50 \\
\hline Hematocrito $(\%)$ & 45 & 36,70 \\
\hline Glucemia (mg/dl) & 88 & 90 \\
\hline Creatinina (mg/dl) & 0,7 & 0,6 \\
\hline $\operatorname{ALT}(\mathrm{U} / \mathrm{L})$ & 19 & 33 \\
\hline AST (U/L) & 22 & 25 \\
\hline GGT/U/L) & 16 & 75 \\
\hline Colesterol total $(\mathrm{mg} / \mathrm{dl})$ & 90 & 170 \\
\hline Triglicéridos (mg/dl) & 33 & 114 \\
\hline $\mathrm{c}-\mathrm{HDL}(\mathrm{mg} / \mathrm{dl})$ & 31 & 24 \\
\hline $\mathrm{c}-\mathrm{LDL}(\mathrm{mg} / \mathrm{dl})$ & 39 & 97 \\
\hline
\end{tabular}


Tabla 2 Manifestaciones demográficas, clínicas y paraclínicas de los dos casos con hipercolesterolemia familiar homocigota

\begin{tabular}{|c|c|c|}
\hline & Caso 1 & Caso 2 \\
\hline \multicolumn{3}{|l|}{ Exámenes- Imágenes } \\
\hline Fracción de eyección (\%) & 65 & 70 \\
\hline Prueba de esfuerzo & Positiva - submáxima & No \\
\hline Angiografía coronaria & Sí & Sí \\
\hline ECG & $\begin{array}{l}\text { Sinusal, patrón bloqueo rama derecha haz de His } \\
\text { Cambios en ST-T }\end{array}$ & Sinusal. Cambios en ST-T \\
\hline \multicolumn{3}{|l|}{ Medicamentos } \\
\hline Ezetimiba & Sí & Sí \\
\hline Simvastatina & Sí & Sí \\
\hline Amlodipino & Sí & No \\
\hline Prednisolona & Sí & No \\
\hline Tacrolimus & Sí & Sí \\
\hline Aspirina & Sí & Sí \\
\hline Clopidogrel & Sí & Sí \\
\hline Valganciclovir & No & Sí \\
\hline Metoprolol & No & Sí \\
\hline \multicolumn{3}{|l|}{ Angiografía coronaria } \\
\hline Lesión ostial & Sí & Sí \\
\hline Calcio & Moderado & Moderado \\
\hline LAD proximal & Sí & No \\
\hline Coronaria derecha & Sí & Sí \\
\hline Circunfleja & No & No \\
\hline $\mathrm{PCI} / \mathrm{DES}$ & Sí -2 vasos & Sí - 1 vaso \\
\hline \multicolumn{3}{|l|}{$\begin{array}{l}\text { Enfermedad vascular } \\
\text { no coronaria }\end{array}$} \\
\hline Carótidas & $30 \%$ & No \\
\hline Aorta & Estenosis supravalvular, placas calcificadas aorta descendente & No \\
\hline Renales & $30 \%$ ostium renal izquierdo & No \\
\hline Miembros inferiores & No & No \\
\hline
\end{tabular}

y $2,25 \times 15 \mathrm{~mm}$ respectivamente, post-dilatación e impactación con balón no complaciente a 16 atms (figs. 3 y 4). En seguimiento clínico a tres meses se le encontró asintomático y adscrito al programa de rehabilitación cardiaca.

\section{Caso 2}

Mujer de 14 años (tablas 1 y 2), con angina de pecho inestable y disnea de inicio reciente y antecedente de HF homocigota (fig. 5). El análisis de historia familiar reveló una historia extensa de HF heterocigota y eventos cardiovasculares en las familias materna y paterna. Se evidenciaron xantomas cutáneos planos y arco corneano (fig. 6), hiperlipidemia severa (c-LDL hasta $644 \mathrm{mg} / \mathrm{dl}$ ) y trasplante de hígado en septiembre de 2013. El análisis genético por secuenciación de los genes LDLR y ApoB identificó una mutación homocigótica o hemicigótica en un intrón de LDLR y una mutación homo- o hemicigótica en un exón de LDLR. Se hallaba en tratamiento con ezetimiba $10 \mathrm{mg} /$ día, simvastatina $20 \mathrm{mg} /$ día, valganciclovir $1 \mathrm{tab} /$ día y tacrolimus $3 \mathrm{mg} /$ día. La angiografía coronaria reveló enfermedad coronaria severa en la coronaria derecha ostial. Se hizo angioplastia coronaria con implante de stent liberador de medicamento Resolute Integrity ${ }^{\top M}$ (Medtronic Vascular, Santa Rosa, CA) $2,5 \times 15 \mathrm{~mm}$ y postdilatación e impactación con balón no complaciente a 16 atms (fig. 7). En el seguimiento clínico a 3 meses se le encontró asintomática y adscrita al programa de rehabilitación cardiaca.

\section{Discusión}

Como se expresó antes, la HF es un trastorno genético autosómico dominante, monogénico, causado por mutaciones en el gen del R-LDL. En este artículo se exponen dos casos con HF homocigota en jóvenes de 14 y 15 años y enfermedad coronaria severa en vasos principales (descendente anterior y coronaria derecha) sometidos a implante exitoso de stent liberador de medicamento. Ambos tenían como característica particular, la edad, las manifestaciones cardiovasculares (angina de pecho) y encontrarse en un periodo postrasplante de hígado ( 3 y 2 meses respectivamente) sin evidencia de rechazo, además de perfil de lípidos en niveles normales.

La literatura describe que en pacientes con HF homocigota, la edad media en el momento del diagnóstico de las enfermedades cardiovasculares es 20 años ${ }^{11,19}$. Sin embargo, varias publicaciones correspondientes a la década de los 80 describen pacientes homocigotos menores de 17 años de edad que desarrollaron enfermedad coronaria severa y estenosis aórtica supravalvular, fecha para cual la terapia de 


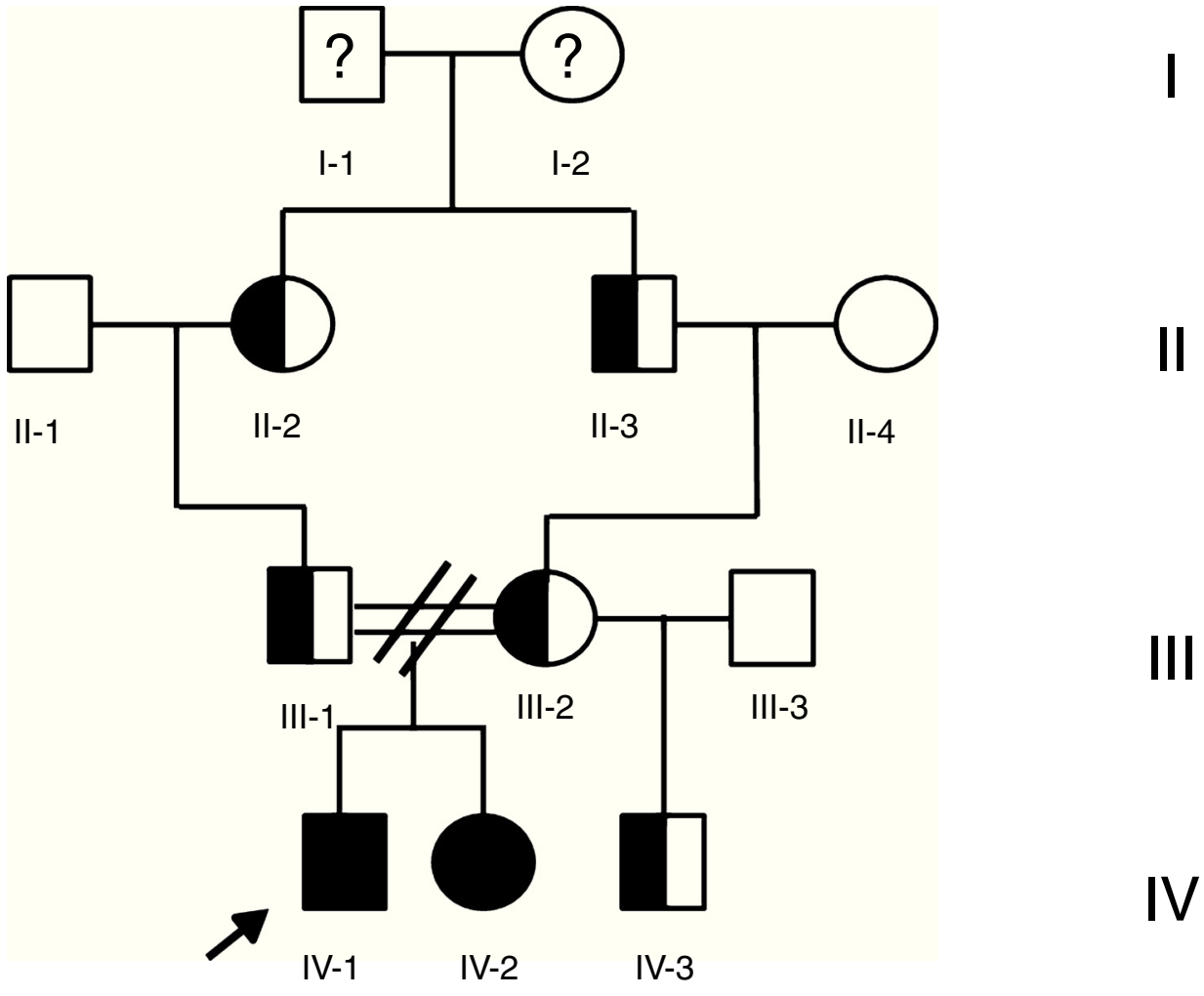

Figura 1 Historia familiar del paciente con hipercolesterolemia homocigota.

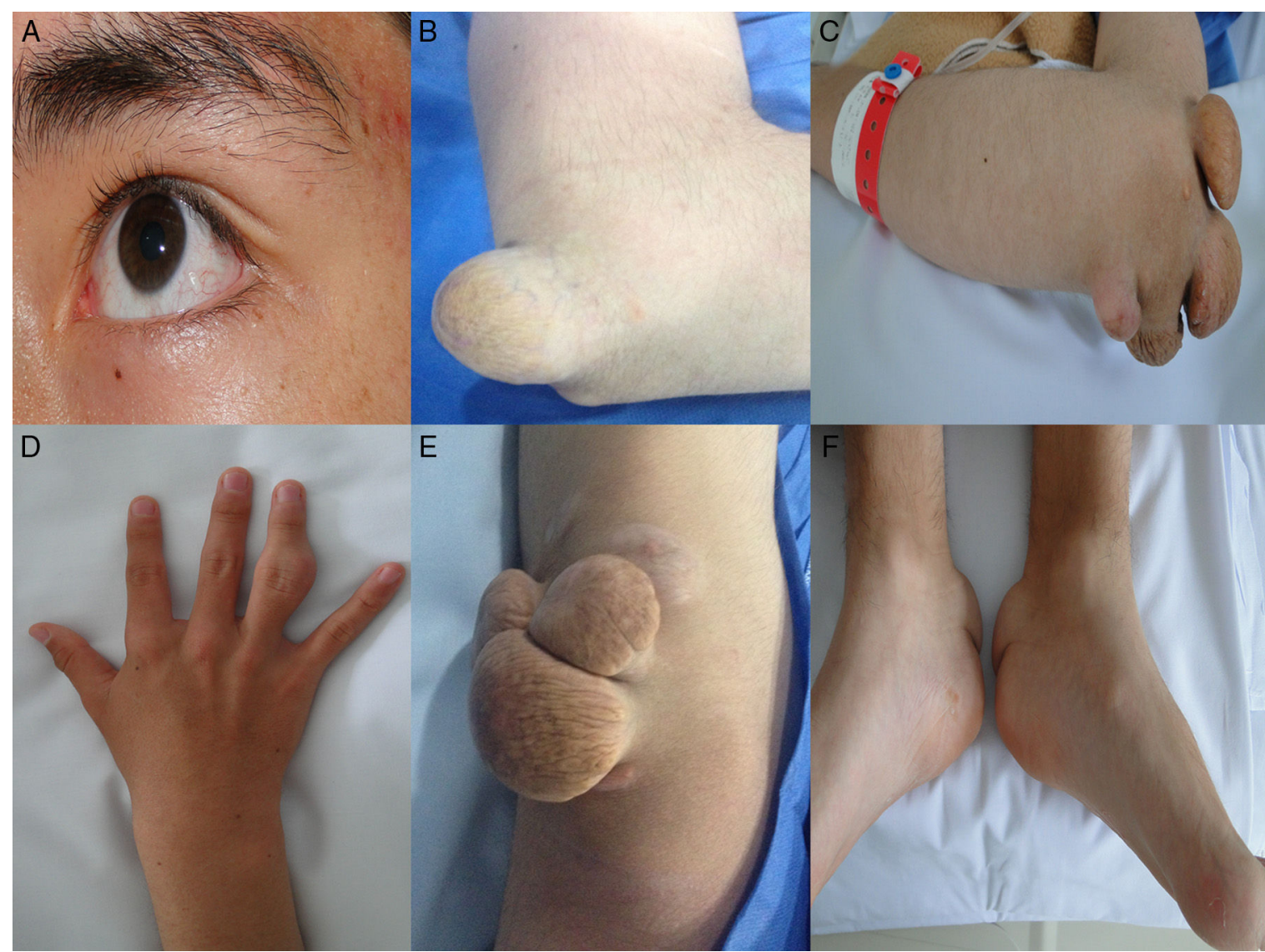

Figura 2 Manifestaciones cutáneas de hipercolesterolemia familiar. Ausencia de arco corneano (A), Xantomas tuberosos en codos $(A$ y $B)$, manos $(C)$, rodillas $(D)$ y tendón de Aquiles $(F)$. 


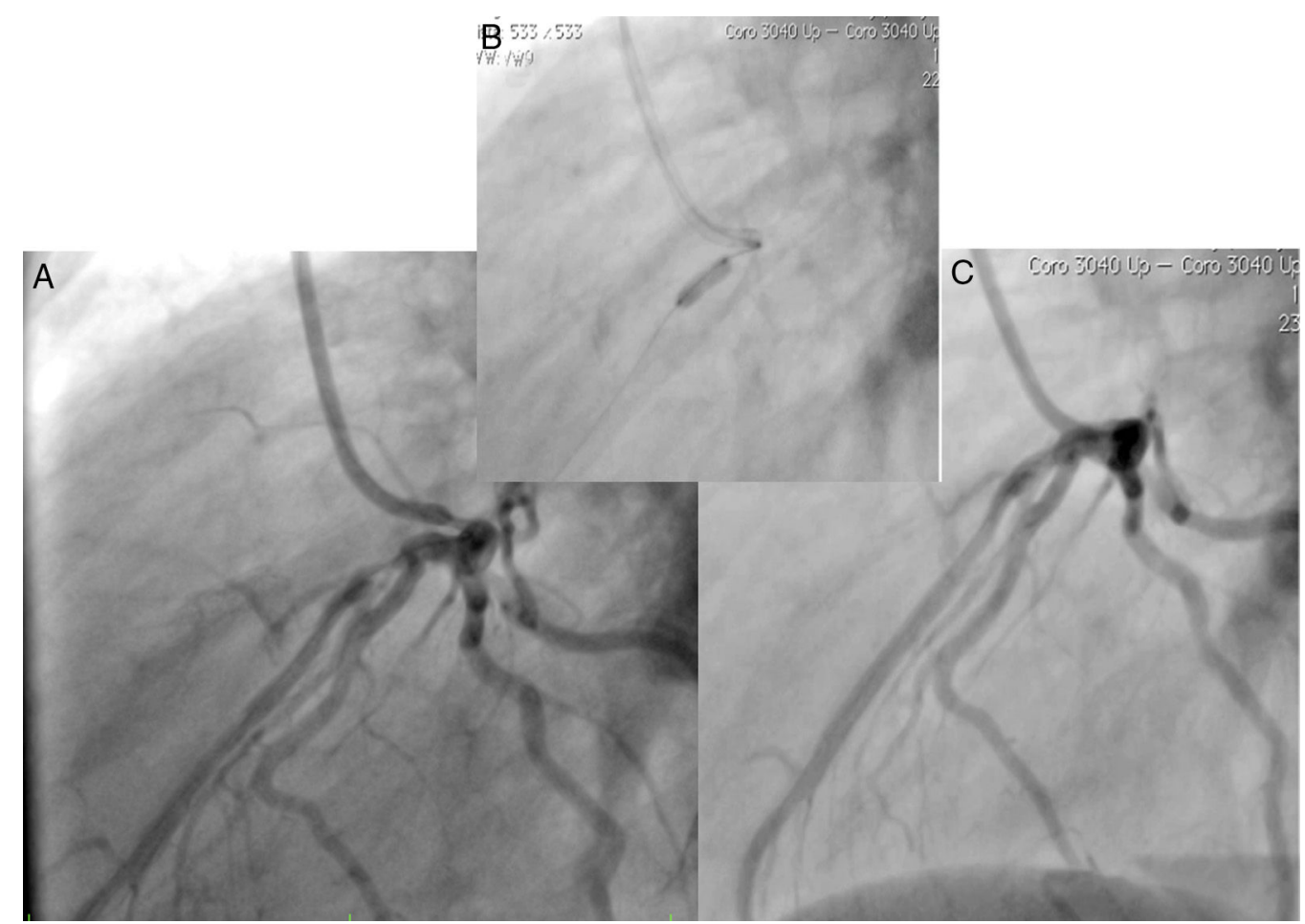

Figura 3 Enfermedad coronaria severa en descendente anterior (A), implante de stent liberador de medicamento (B) y resultado final (C).

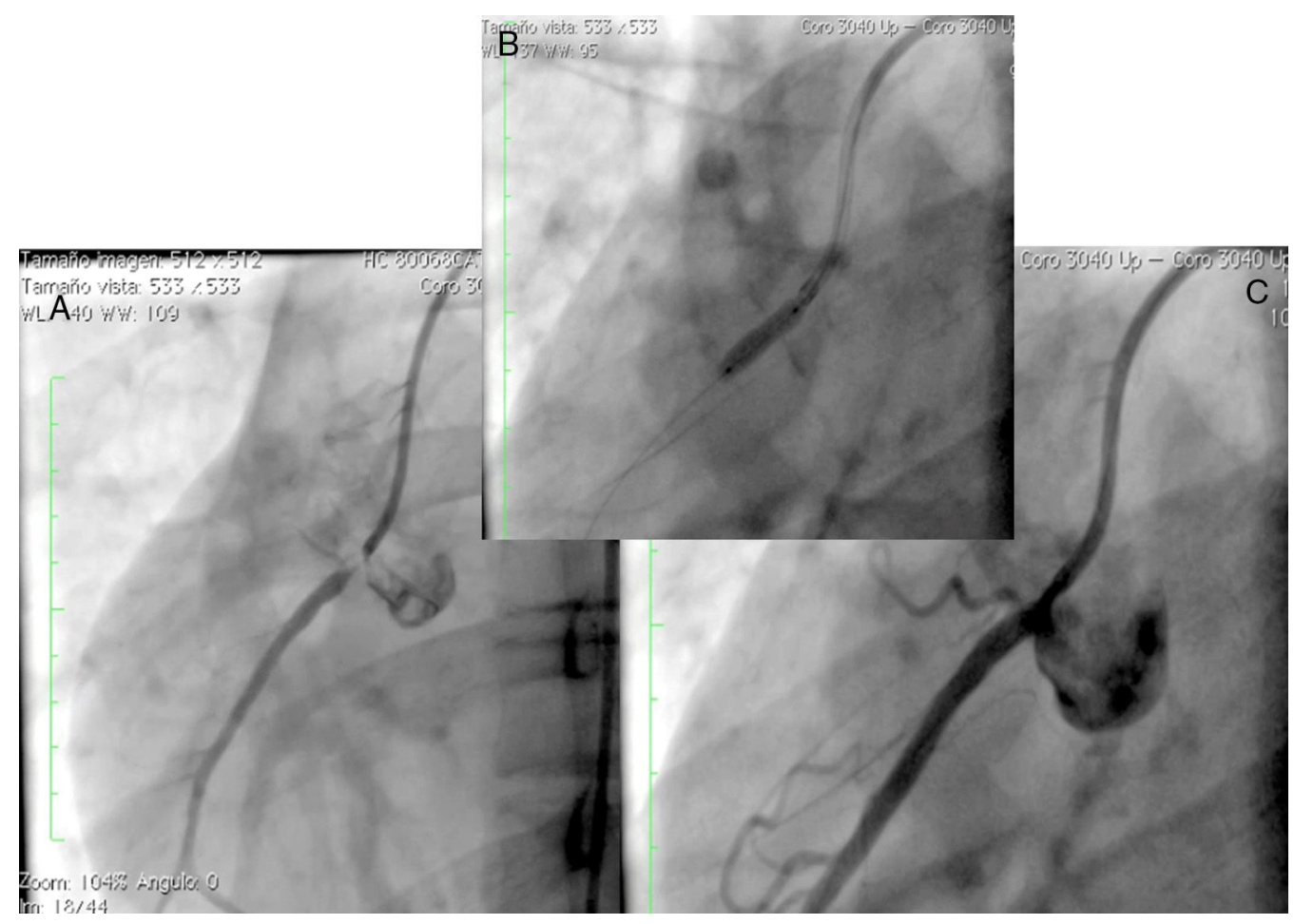

Figura 4 Enfermedad coronaria severa en coronaria derecha ostial (A), implante de stent liberador de medicamento (B) y resultado final (C). 


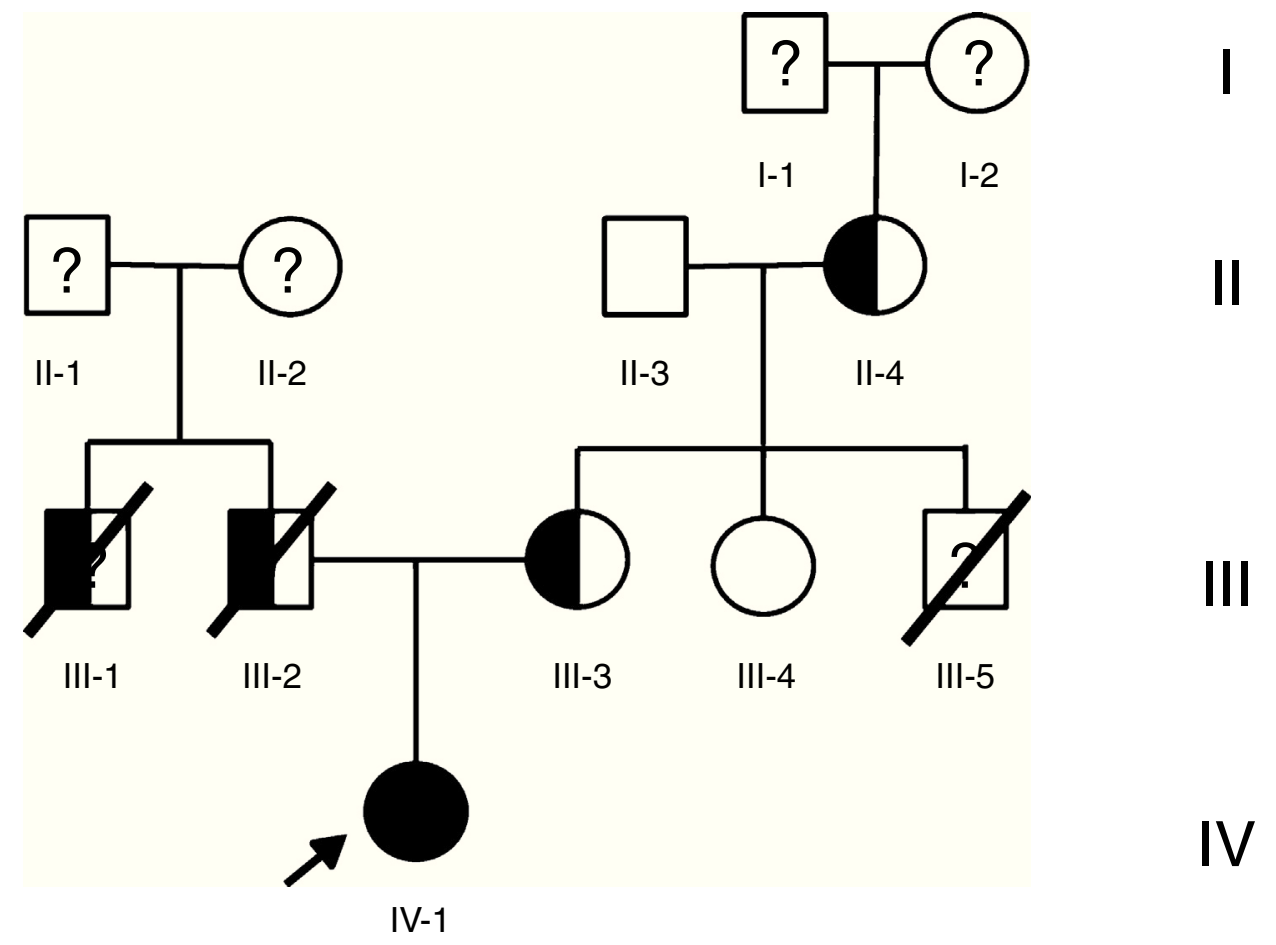

Figura 5 Historia familiar de la paciente con hipercolesterolemia familiar homocigota.

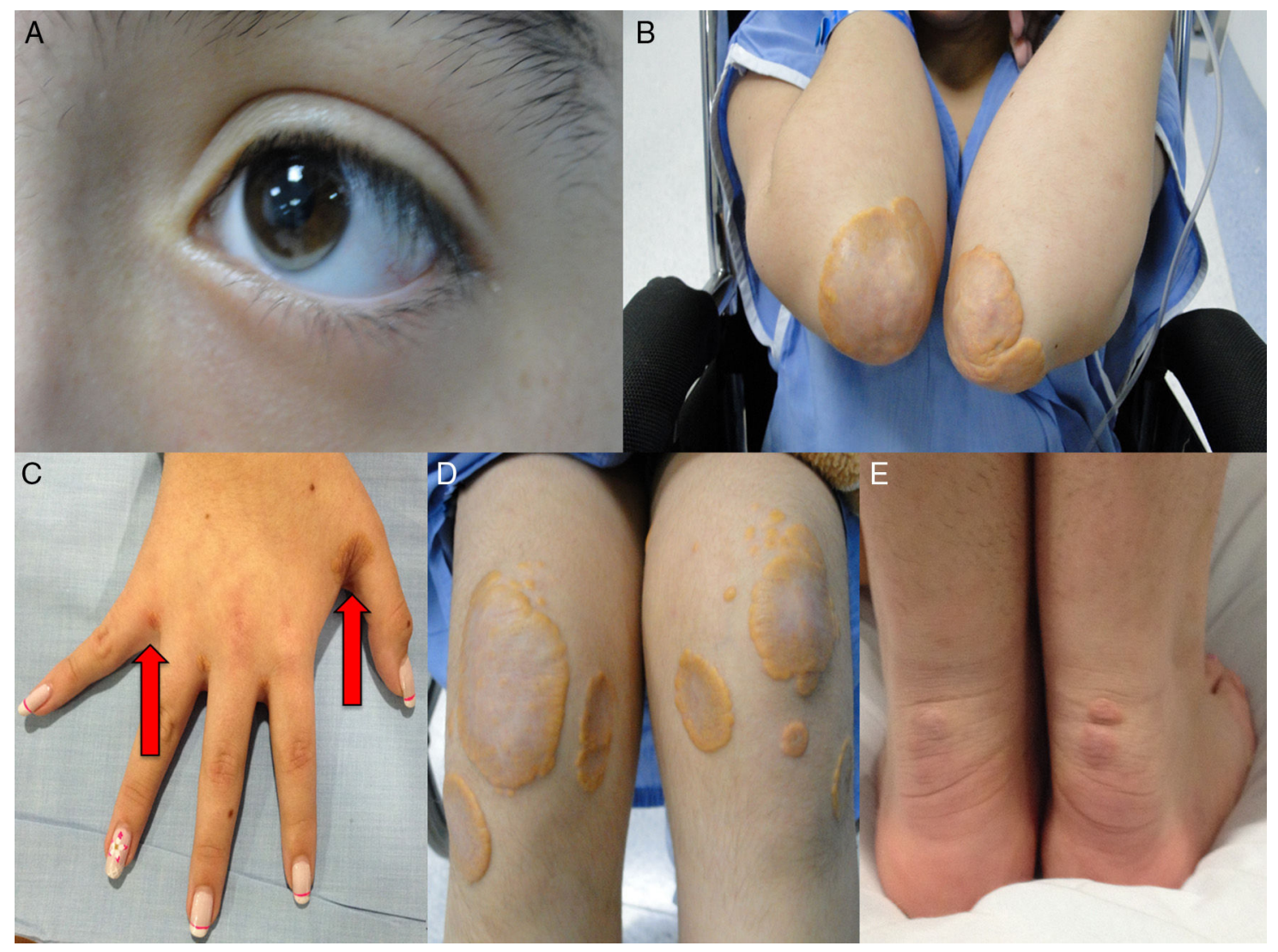

Figura 6 Manifestaciones cutáneas de hipercolesterolemia familiar. Arco corneano inferior (A), Xantomas planos en codos (A), manos $(C)$, rodillas $(D)$ y tendón de Aquiles $(E)$. 


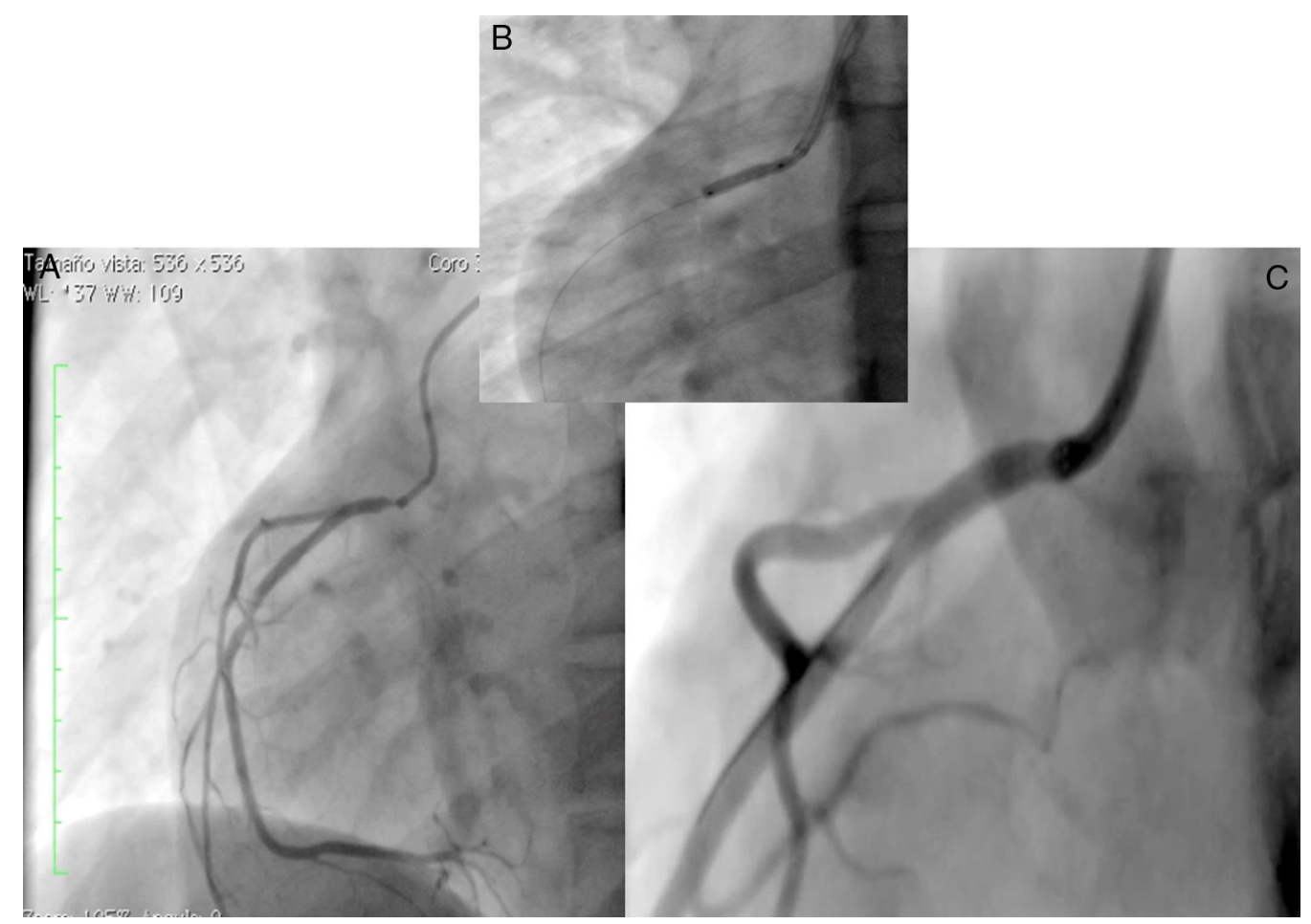

Figura 7 Enfermedad coronaria severa en coronaria derecha ostial (A), implante de stent liberador de medicamento (B) y resultado final (C).

revascularización coronaria percutánea con stents estaba en sus inicios $2,15,20,21$.

Tellez et al. ${ }^{22}$ describieron en 2010 la respuesta vascular que ocurre después del implante de un stent metálico en un modelo porcino con HF, demostrando un comportamiento en la formación neointimal más agresivo que el que ocurre en animales domésticos, lo cual plantea la necesidad de considerar el uso de stents liberadores de medicamento en condiciones de HF. Recientemente, Shankarappa et $\mathrm{al}^{14}$ reportaron los casos de 5 pacientes, 4 hombres y 1 mujer, tratados en el Instituto Sri Jayadeva de Cardiología, en Bangalore, India. Todos presentaron síntomas coronarios, xantomas tendinosos y enfermedad coronaria severa, además de cifras de colesterol total mayores a $435 \mathrm{mg} / \mathrm{dl}$ y c-LDL mayores a $392 \mathrm{mg} / \mathrm{dl}$. Tres de ellos requirieron cirugía de revascularización arterial y los otros dos, angioplastia con implante exitoso de stents. En un hombre de 24 años se implantó stent liberador de medicamento en descendente anterior y stent metálico en circunfleja, y en una mujer de 17 años stent liberador de medicamento en tronco principal izquierdo.

La intervención coronaria percutánea (ICP) se utiliza comúnmente en pacientes adultos con enfermedad coronaria severa. En aquellos con angina inestable o infarto sin elevación del ST, se indica estrategia invasiva precoz (clase I, nivel de evidencia $B)^{23}$. De otra parte, la información de ICP en niños y adolescentes es muy limitada. La mayoría de los casos descritos en menores de 18 años, han tenido indicación no aterosclerótica, como vasculopatía coronaria del trasplante cardiaco ${ }^{24-27}$, disección coronaria ${ }^{24}$ y compresión u oclusión coronaria como secuela de cirugía de corazón previa $^{24,28,29}$.
Las Guías publicadas por la US National Lipid Association (NLA) y NICE en el Reino Unido, recomiendan una reducción en la concentración de C-LDL de más del $50 \%$ respecto a los niveles previos al tratamiento en pacientes con $\mathrm{HF}^{30-33}$. Las Guías de Canadá y Europa recomiendan la reducción de los niveles de c-LDL a menos de $116 \mathrm{mg} / \mathrm{dl}$ en pacientes con riesgo moderado para enfermedad cardiovascular; menos de $97 \mathrm{mg} / \mathrm{dl}$ en pacientes de alto riesgo, y menos de $70 \mathrm{mg} / \mathrm{dl}$ en pacientes de muy alto riesgo.

Hoy día se dispone de diferentes opciones para el tratamiento de los pacientes afectados por HF, entre ellas, dieta, terapia farmacológica, aféresis de lípidos y algunas técnicas quirúrgicas como la cirugía de derivación portocava que limita la absorción de colesterol y promueve la pérdida de ácidos biliares, y el trasplante de hígado; para disponer de Rs-LDL funcionales, este último constituye una alternativa para los casos más graves ${ }^{34}$.

Pocos años después que Brown y Goldstein ganaran el premio Nobel de Medicina en 1985 al describir que la HF se debía a un defecto intrínseco del hepatocito en la deficiencia de receptores de c-LDL como explicación de las cifras extremadamente altas de colesterol sérico ${ }^{21}$, Starzl et al. ${ }^{35}$ reportaron el primer trasplante hepático ortotópico realizado fuera de las indicaciones habituales de una insuficiencia hepática, como tratamiento de la hiperlipidemia en el contexto de HF homocigótica. Fue la primera vez que un hígado anatómicamente normal se extirpaba quirúrgicamente en una paciente de seis años de edad, para el tratamiento presuntivo de la condición hipercolesterolémica.

Como bien se sabe, la mayoría de los R-LDL se encuentran en el hígado, por ello el trasplante de hígado se ha 
convertido en el tratamiento de elección para los pacientes afectados que no responden a tratamientos farmacológicos de rutina ${ }^{36,37}$. El hígado trasplantado conserva las cualidades específicas del donante, de modo que el trasplante puede ser fuente abundante de receptores de LDL funcionales, y conducir a la cura de la hipercolesterolemia ${ }^{34}$. Gracias a los avances en la experiencia por parte de los grupos de trasplante, la inmunosupresión a largo plazo, mejoras en las técnicas quirúrgicas y los métodos inmunológicos, se han alcanzado resultados favorables después del trasplante tanto en población adulta como pediátrica ${ }^{35,38}$. Los casos expuestos tenían al momento de su intervención coronaria, trasplante hepático exitoso y cifras de colesterol normales.

\section{Conclusiones y recomendaciones}

La HF es un trastorno genético autosómico dominante, asociado con niveles elevados de c-LDL, que puede conducir a enfermedad cardiovascular prematura. El diagnóstico precoz es crucial para prevenir la morbilidad y la mortalidad. Este generalmente se hace con base en las características clínicas, los antecedentes familiares y los niveles séricos de colesterol. Las guías actuales destacan la importancia de reducir los niveles de c-LDL en pacientes con HF. En este documento se informaron los casos de dos pacientes de 14 y 15 años con manifestaciones clínicas de HF homocigota severa tratada con fármacos y trasplante hepático, que además presentaron síndrome coronario agudo sin elevación del ST y enfermedad coronaria severa tratada de manera exitosa con implante de stents liberadores de medicamento.

\section{Responsabilidades éticas}

Protección de personas y animales. Los autores declaran que para esta investigación no se han realizado experimentos en seres humanos ni en animales.

Confidencialidad de los datos. Los autores declaran que han seguido los protocolos de su centro de trabajo sobre la publicación de datos de pacientes.

Derecho a la privacidad y consentimiento informado. Los autores han obtenido el consentimiento informado de los pacientes y/o sujetos referidos en el artículo. Este documento obra en poder del autor de correspondencia.

\section{Conflicto de interés}

Los autores declaran no tener conflictos de interés.

\section{Bibliografía}

1. Go AS, Mozaffarian D, Roger VL, Benjamin EJ, Berry JD, Blaha MJ, et al. Heart disease and stroke statistics-2014 Update. Circulation. 2014;129:e28-92.

2. Sprecher DL, Schaefer EJ, Kent KM, Gregg RE, Zech LA, Hoeg $J M$, et al. Cardiovascular features of homozygous familial hypercholesterolemia: analysis of 16 patients. Am J Cardiol. 1984;54:20-30.

3. Hopkins PN, Toth PP, Ballantyne CM, Rader DJ. National Lipid Association Expert Panel on Familial Hypercholesterolemia.
Familial hypercholesterolemias: prevalence, genetics, diagnosis and screening recommendations from the National Lipid Association Expert Panel on Familial Hypercholesterolemia. J Clin Lipidol. 2011;5 3 Suppl:S9-17.

4. Liyanage KE, Burnett JR, Hooper AJ, van Bockxmeer FM. Familial hypercholesterolemia: epidemiology. Neolithic origins and modern geographic distribution. Crit Rev Clin Lab Sci. 2011;48:1-18.

5. Goldberg AC, Hopkins PN, Toth PP, Ballantyne CM, Rader DJ, Robinson JG, et al. Familial hypercholesterolemia: screening, diagnosis and management of pediatric and adult patients: clinical guidance from the National Lipid Association Expert Panel on Familial Hypercholesterolemia. J Clin Lipidol. 2011;5 3 Suppl:S1-8.

6. Wierzbicki AS, Humphries SE, Minhas R. Guideline Development Group. Familial hypercholesterolaemia: summary of NICE guidance. BMJ. 2008;337:a1095.

7. Hobbs HH, Brown MS, Russell DW, Davignon J, Goldstein JL. Deletion in the gene for the low-density-lipoprotein receptor in a majority of French Canadians with familial hypercholesterolemia. N Engl J Med. 1987;317:734-7.

8. Moorjani S, Roy M, Gagné C, Davignon J, Brun D, Toussaint M, et al. Homozygous familial hypercholesterolemia among French Canadians in Québec Province. Arteriosclerosis. 1989;9:211-6.

9. Yuan G, Wang J, Hegele RA. Heterozygous familial hypercholesterolemia: an underrecognized cause of early cardiovascular disease. CMAJ. 2006;174:1124-9.

10. Zeegers MPA, van Poppel F, Vlietinck R, Spruijt L, Ostrer $\mathrm{H}$. Founder mutations among the Dutch. Eur J Hum Genet. 2004; 12:591-600.

11. Kolansky DM, Cuchel M, Clark BJ, Paridon S, MCCrindle BW, Wiegers SE, et al. Longitudinal evaluation and assessment of cardiovascular disease in patients with homozygous familial hypercholesterolemia. Am J Cardiol. 2008;102:1438-43.

12. Naoumova RP, Thompson GR, Soutar AK. Current management of severe homozygous hypercholesterolaemias. Curr Opin Lipidol. 2004; 15:413-22.

13. Liyanage KE, Hooper AJ, Defesche JC, Burnett JR, van Bockxmeer FM. High-resolution melting analysis for detection of familial ligand-defective apolipoprotein B-100 mutations. Ann Clin Biochem. 2008;45 Pt 2:170-6.

14. Shankarappa RK, Moorthy N, Bhat SPS, Dwarakaprasad R, Nanjappa MC. The challenge produced by familial homozygous hypercholesterolemia when treating premature coronary arterial disease in the young. Cardiol Young. 2009;19:257-63.

15. Allen JM, Thompson GR, Myant NB, Steiner R, Oakley CM. Cadiovascular complications of homozygous familial hypercholesterolaemia. Br Heart J. 1980;44:361-8.

16. Hutter CM, Austin MA, Humphries SE. Familial hypercholesterolemia, peripheral arterial disease, and stroke: a HuGE minireview. Am J Epidemiol. 2004;160:430-5.

17. Civeira F, Castillo S, Alonso R, Meriño-Ibarra E, Cenarro A, Artied $M$, et al. Tendon xanthomas in familial hypercholesterolemia are associated with cardiovascular risk independently of the low-density lipoprotein receptor gene mutation. Arterioscler Thromb Vasc Biol. 2005;25:1960-5.

18. Merchán A, Ruiz A, Campo R, Prada C, Toro J, Sánchez R, et al. Hipercolesterolemia familiar: artículo de revisión. Rev Col Cardiol. 2016;23 Supl 4:4-26.

19. Robinson JG. Management of familial hypercholesterolemia: a review of the recommendations from the National Lipid Association Expert Panel on Familial Hypercholesterolemia. J Manag Care Pharm. 2013;19:139-49.

20. Mabuchi H, Koizumi J, Shimizu M, Takeda R. Development of coronary heart disease in familial hypercholesterolemia. Circulation. 1989;79:225-32.

21. Goldstein JL, Brown MS. Familial hypercholesterolemia. En: Stanbury JB, Wyngaarden JB, Fredrickson DS, Goldstein JL, 
Brown MS, editores. The Metabolic Basis of Inherited Disease. 5 ed. New York: McGraw-Hill Book Co; 1983. p. 672.

22. Tellez A, Krueger CG, Seifert P, Winsor-Hines D, Piedrahita C, Cheng $Y$, et al. Coronary bare metal stent implantation in homozygous LDL receptor deficient swine induces a neointimal formation pattern similar to humans. Atherosclerosis. 2010;213:518-24.

23. Levine GN, Bates ER, Blankenship JC, Bailey SR, Bittl JA, Cercek B, et al. 2011 ACCF/AHA/SCAI Guideline for Percutaneous Coronary Intervention: a report of the American College of Cardiology Foundation/American Heart Association Task Force on Practice Guidelines and the Society for Cardiovascular Angiography and Interventions. Circulation. 2011;124:e574-651.

24. Schneider AE, Johnson JN, Taggart NW, Cabalka AK, Hagler DJ, Reeder GS, et al. Percutaneous coronary intervention in pediatric and adolescent patients. Congenit Heart Dis. 2014;9:228-34.

25. Lee MS, Sachdeva R, Kim MH, Sachdeva R. Long-term outcomes of percutaneous coronary intervention in transplant coronary artery disease in pediatric heart transplant recipients. J Invasive Cardiol. 2012;24:278-81.

26. Sachdeva R, Seib PM, Frazier EA, Sachdeva R. Percutaneous coronary intervention using drug-eluting stents in pediatric heart transplant recipients. Pediatr Transplant. 2009;13:1014-9.

27. Salloum JG, Dodd DA, Slosky D, Zhao DXM. Treatment of unprotected left main coronary artery stenosis in a 5-year-old heart transplant patient using a sirolimus-eluting stent. J Heart Lung Transplant. 2007;26:1061-4.

28. Natal-Hernandez L, Meadows J, Shunk KA, Boyle AJ. Percutaneous retrograde recanalization of a chronic total coronary artery occlusion in a 7 year old. Cardiovasc Revasc Med. 2013;14:113-7.

29. Kaichi S, Doi H, Tsurumi F, Heike T. Long-term outcome of sirolimus-eluting stent implantation for left main coronary artery stenosis in infancy. Pediatr Cardiol. 2011;32:94-7.

30. Goldberg AC, Hopkins PN, Toth PP, Ballantyne CM, Rader DJ, Robinson JG, et al. Familial hypercholesterolemia: screening, diagnosis and management of pediatric and adult patients: clinical guidance from the National Lipid Association Expert Panel on Familial Hypercholesterolemia. J Clin Lipidol. 2011;5: 133-40.

31. Reiner Z, Catapano AL, De Backer G, Graham I, Taskinen MR, Wiklund O, et al. European Association for Cardiovascular Prevention \& Rehabilitation ESC/EAS Guidelines for the management of dyslipidaemias: the Task Force for the management of dyslipidaemias of the European Society of Cardiology (ESC) and the European Atherosclerosis Society (EAS). Eur Heart J. 2011;32:1769-818.

32. Grundy SM, Cleeman JI, Merz CNB, Brewer HB, Clark LT, Hunninghake DB, et al. Implications of recent clinical trials for the National Cholesterol Education Program Adult Treatment Panel III Guidelines. J Am Coll Cardiol. 2004;44:720-32.

33. Genest J, McPherson R, Frohlich J, Anderson T, Campbell N, Carpentier A, et al. 2009 Canadian Cardiovascular Society/Canadian guidelines for the diagnosis and treatment of dyslipidemia and prevention of cardiovascular disease in the adult - 2009 recommendations. Can J Cardiol. 2009;25:567-79.

34. Nemati MH, Astaneh B. Optimal management of familial hypercholesterolemia: treatment and management strategies. Vasc Health Risk Manag. 2010;6:1079-88.

35. Maiorana A, Nobili V, Calandra S, Francalanci P, Bernabei S, El Hachem $M$, et al. Preemptive liver transplantation in a child with familial hypercholesterolemia. Pediatr Transplant. 2011;15:E25-9.

36. Moghadasian MH, Frohlich JJ, Saleem M, Hong JM, Qayumi K, Scudamore $\mathrm{CH}$. Surgical management of dyslipidemia: clinical and experimental evidence. J Invest Surg. 2001;14:71-8.

37. Bilheimer DW, Goldstein JL, Grundy SM, Starzl TE, Brown MS. Liver transplantation to provide low-density-lipoprotein receptors and lower plasma cholesterol in a child with homozygous familial hypercholesterolemia. N Engl J Med. 1984;311:1658-64.

38. López-Santamaria M, Migliazza L, Gamez M, Murcia J, DiazGonzalez M, Camarena C, et al. Liver transplantation in patients with homozygotic familial hypercholesterolemia previously treated by end-to-side portocaval shunt and ileal bypass. J Pediatr Surg. 2000;35:630-3. 